\title{
Understanding Low Reliability of Memories for Neutral Information Encoded under Stress: Alterations in Memory-Related Activation in the Hippocampus and Midbrain
}

\author{
Shaozheng Qin, ${ }^{1,2}$ Erno J. Hermans, ${ }^{1,3}$ Hein J. F. van Marle, ${ }^{1,4}$ and Guillén Fernández ${ }^{1,3}$ \\ ${ }^{1}$ Radboud University Nijmegen, Donders Institute for Brain, Cognition and Behaviour, 6500 HC Nijmegen, the Netherlands, ${ }^{2}$ Department of Psychiatry and \\ Behavioral Sciences, Stanford University School of Medicine, Stanford, California 94304, ${ }^{3}$ Radboud University Nijmegen Medical Center, Department for \\ Cognitive Neuroscience, 6500 HC Nijmegen, the Netherlands, and ${ }^{4}$ Department of Psychiatry, Academic Medical Center, University of Amsterdam, 1012 \\ WX Amsterdam, the Netherlands
}

\begin{abstract}
Exposure to an acute stressor can lead to unreliable remembrance of intrinsically neutral information, as exemplified by low reliability of eyewitness memories, which stands in contrast with enhanced memory for the stressful incident itself. Stress-sensitive neuromodulators (e.g., catecholamines) are believed to cause this low reliability by altering neurocognitive processes underlying memory formation. Using event-related functional magnetic resonance imaging, we investigated neural activity during memory formation in 44 young, healthy human participants while incidentally encoding emotionally neutral, complex scenes embedded in either a stressful or neutral context. We recorded event-related pupil dilation responses as an indirect index of phasic noradrenergic activity. Autonomic, endocrine, and psychological measures were acquired to validate stress manipulation. Acute stress during encoding led to a more liberal response bias (more hits and false alarms) when testing memory for the scenes $24 \mathrm{~h}$ later. The strength of this bias correlated negatively with pupil dilation responses and positively with stress-induced heart rate increases at encoding. Acute stress, moreover, reduced subsequent memory effects (SMEs; items later remembered vs forgotten) in hippocampus and midbrain, and in pupil dilation responses. The diminished SMEs indicate reduced selectivity and specificity in mnemonic processing during memory formation. This is in line with a model in which stress-induced catecholaminergic hyperactivation alters phasic neuromodulatory signaling in memory-related circuits, resulting in generalized (gist-based) processing at the cost of specificity. Thus, one may speculate that loss of specificity may yield less discrete memory representations at time of encoding, thereby causing a more liberal response bias when probing these memories.
\end{abstract}

\section{Introduction}

Stress has a powerful impact on learning and memory. Whereas exposure to an acute stressor can enhance emotional aspects of episodic memories, memory for intrinsically neutral information encountered within the same context becomes unreliable (Payne et al., 2006). For instance, eyewitness testimony often proves untrustworthy (Loftus, 1979), and studies have shown increased false positives under stress (Payne et al., 2002). Psychological views hold that these phenomena result from a stressinduced shift toward generalized processing, such as extracting central thematic information (gist) at the cost of specificity

\footnotetext{
Received June 17, 2011; revised Jan. 25, 2012; accepted Feb. 6, 2012

Author contributions: S.Q., E.J.H., H.J.F.v.M., and G.F. designed research; S.Q. performed research; S.Q. and H.J.F.v.M. contributed unpublished reagents/analytic tools; S.Q. analyzed data; S.Q., E.J.H., and G.F. wrote the paper. This work was supported by the Netherlands Organization for Scientific Research Grants 918.66.613, 451.07.019, and 446.10 .010 .

Correspondence should be addressed to Dr. Shaozheng Qin, Department of Psychiatry and Behavioral Sciences, Stanford University School of Medicine, 1070 Arastradero Road, Ste 220, Palo Alto, CA 94304. E-mail: szqin@stanford.edu.

DOI:10.1523/JNEUROSCI.3101-11.2012

Copyright $\odot 2012$ the authors $\quad 0270-6474 / 12 / 324032-10 \$ 15.00 / 0$
}

(Payne et al., 2002). Such a shift has been related to stress-induced alterations in neurocognitive processes underlying memory (Christianson, 1992) through hyperactivation of stress-sensitive neuromodulatory (e.g., catecholaminergic) systems (Aston-Jones and Cohen, 2005). However, it remains open how acute stress affects the neural basis underlying memory formation, particularly for neutral information encountered in a stressful context.

The formation of new memories, supported by the hippocampus, among other regions, is modulated by noradrenergic and dopaminergic systems in the midbrain (Lisman and Grace, 2005; Sara, 2009). In an attentive state, these systems facilitate hippocampal functioning through increased stimulus-related phasic firing patterns, thereby promoting the formation of clean, discrete memory representations. In agreement with this notion, neuroimaging studies have found robust subsequent memory effects (SMEs; stronger phasic neural responses for later remembered than forgotten items) in hippocampus and in midbrain regions where catecholaminergic nuclei are located (Schott et al., 2006; Shohamy and Wagner, 2008).

Exposure to an acute stressor leads not only to activation of the sympathetic nervous system (SNS) and the hypothalamic- 


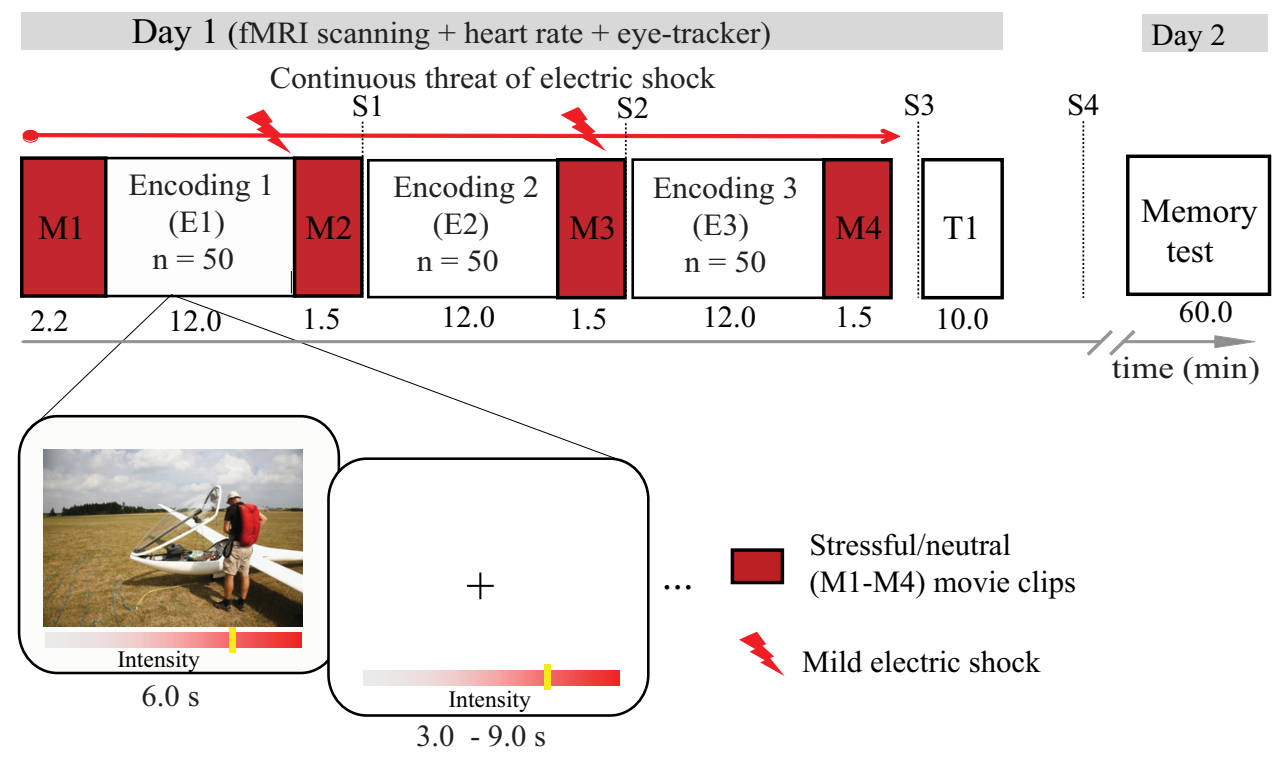

Figure 1. Experimental design. On Day 1, after a period of preparation and training, participants were scanned while performing an incidental memory encoding task that was fully embedded in either an acutely stressful or an emotionally neutral context. Stress induction was implemented using short aversive movie clips and combined with continuous threat of (mild) electrical shock with gradually increasing intensity. The experiment started with the first movie clip (M1) at time point 0 in the scanner and was followed by a first encoding run (E1), a second movie clip (M2), a second encoding run (E2), a third movie clip (M3), a third encoding run (E3), a forth movie clip (M4), and a structural scan (T1). Continuous threat of mild electrical shock was present throughout scanning, with gradually increasing intensity (as indicated by a bar at bottom of the screen). In the control condition, the aversive clips were replaced by neutral movie fragments, and no threat of shock was present. Participants were told that intensity bar was implemented for monitoring the progress of the experiment. Before, in between, and during fMRI scanning, salivary samples, heart rate, pupil diameter (eye-tracking), and subjective mood state were obtained to assess the effectiveness of stress induction. A memory test was administered the following day. For more details, see Materials and Methods. n, Number of pictures in each encoding run.

pituitary-adrenal (HPA) axis, but also to hyperactivation of central catecholaminergic systems (de Kloet et al., 2005). For instance, the locus ceruleus-centered norepinephrine (LC$\mathrm{NE})$ system has been shown to shift into a mode with tonically elevated background activity, but diminished stimulus-related phasic firing (Valentino and Van Bockstaele, 2008). Neural responses to sensory stimuli consequently become less selective and more generalized, creating a hypervigilant state (Aston-Jones and Cohen, 2005). In humans, it has been shown that stimulus-related phasic pupil responses, which closely parallel LC-NE activity (Bradley et al., 2008; Gilzenrat et al., 2010), are diminished under acute stress (Henckens et al., 2009). Thus, one may conjecture that stress-induced catecholaminergic activity shifts neuronal processing into a mode with high tonic but reduced phasic responses, resulting in altered neural activity and pupil responses related to memory formation.

To investigate this, 44 young healthy participants underwent event-related functional magnetic resonance imaging (fMRI) and continuous pupillometry during incidental encoding of complex scenes depicting meaningful, emotionally neutral activities. Acute stress was experimentally induced in half of the participants by showing aversive movie clips with self-referencing instructions, in conjunction with continuous threat of electrical shock. Twenty-four hours later, a surprise memory test was performed using short, written descriptions of studied scenes. Subsequent memory analyses for fMRI and pupil data were used to localize and quantify SMEs reflecting phasic neural and pupil responses associated with memory formation. We predicted that acute stress would alter SMEs in memory-related neural circuits and in pupil dilation responses.

\section{Materials and Methods}

\section{Participants}

Forty-four young, healthy, male volunteers (aged 19-36 years) with normal or corrected-to-normal vision participated in this study. Participants reported no history of neurological, psychiatric, or endocrine disease, no current use of any psychoactive drugs or corticosteroids, and no habit of watching violent movies or playing violent video games. None of them had experienced severe physical or emotional trauma. To avoid gender differences (Kudielka and Kirschbaum, 2005) and menstrual cycle effects (Ossewaarde et al., 2010) in stress responsiveness, only men were included. Ethical approval was obtained from the local institutional review board (CMO Region Arnhem-Nijmegen, The Netherlands) and all participants gave written informed consent before the experiment.

Participants were randomly assigned to either the stress induction ( $n=22$; aged $21.65 \pm 3.73$ years) or the control group ( $n=22$; aged $22.71 \pm 4.01$ years). There was no difference in age between the two groups $(t<1)$. Data from four participants were excluded from further analyses (two in each group) due to either poor memory performance or excessive head movement during scanning.

\section{General procedure}

The experiment was performed between 2:00 and 8:00 P.M. to ensure relatively stable and low levels of endogenous cortisol. To reduce anticipation of stress induction for participants in the neutral group, they were told which of the two experimental groups they were assigned to $1 \mathrm{~d}$ before the experiment. Participants were restricted from food or drink intake at least $2 \mathrm{~h}$ before the experiment. After arrival, $1.5 \mathrm{~h}$ before entering the MR scanner, they completed personality questionnaires, trained in the memory encoding task, and prepared for heart rate (HR) measurement. The actual fMRI experiment consisted of three runs of an incidental encoding task that were fully embedded in either a continuously stressful or an emotionally neutral control context. The three encoding runs were separated by four short movie clips to boost stress induction. The experiment ended with a structural scan (Fig. 1). 
Table 1. Grouping of encoding trials based on subsequent memory performance

\begin{tabular}{|c|c|c|c|c|c|}
\hline & \multirow[b]{2}{*}{ Misses } & \multicolumn{4}{|c|}{ Hits: four levels of confidence } \\
\hline & & Very unsure & $\begin{array}{l}\text { Somewhat } \\
\text { unsure }\end{array}$ & $\begin{array}{l}\text { Somewhat } \\
\text { sure }\end{array}$ & Very sure \\
\hline \multirow{3}{*}{$\begin{array}{l}\text { Stress }(n=20) \\
\text { Control }(n=20) \\
\text { Regressors }\end{array}$} & $38.9(2.97)$ & $17.24(2.48)$ & $17.05(1.36)$ & $14.57(1.56)$ & $62.29(4.09)$ \\
\hline & $50.65(2.97)$ & $14.70(1.91)$ & $14.10(0.81)$ & $10.70(1.14)$ & $59.45(4.22)$ \\
\hline & Forgotten & \multicolumn{3}{|c|}{ Low confidence } & Remembered \\
\hline
\end{tabular}

Hits that received "very sure" confidence ratings were grouped as later remembered, and misses were grouped as later forgotten. Remaining (low confidence hit) trials were modeled as a regressor of no interest in the fMRI analysis.

\section{Stress induction}

Acute psychological stress was induced by showing strongly aversive movie clips with a self-referencing instruction in the scanner (Qin et al., 2009; van Marle et al., 2009), combined with continuous threat of (mild) electrical shock (Hermans et al., 2006). Stress-induction movie clips contained scenes with extreme male-to-male aggressive behavior and violence in front of a crowd, selected from a commercial movie (Irreversible, 2002, by Gaspar Noé). After short introductory texts, participants were asked to constantly and attentively view the clips and project themselves into the scene from an eye-witness perspective, thus attempting to involve them maximally in the experience. Participants were told that they would receive a random number of shocks during the encoding session, and that subsequent shocks would increase in strength and latency. To visualize the increase in the strength of shocks, an analog scale indicating a gradually increasing intensity of electrical shocks was always presented at the bottom of the screen. In reality, only two mild electrical shocks were given at fixed time points: one during the last three encoding trials in the first encoding block, and one during the third movie clip. The electrical shocks, generated by a $9 \mathrm{~V}$ batteryoperated device (Tens Elpha 2000; Danmeter), were delivered transcutaneously over the volunteers' left index and middle fingertips using $\mathrm{Ag} / \mathrm{AgCl}$ electrodes.

In the control group, participants watched equally long movie clips selected from another movie (Comment j'ai tué mon père, 2001, by Anne Fontaine), and no shocks were given. Matching for audiovisual characteristics was performed in a separate pilot study by selecting aversive and neutral movie clips out of a set of candidate clips. Two types of movie clips best matched on the following measures: presence of faces in the foreground, presence of background actors, amount of distinct camera movements, and percentage of time the camera was moving (Hermans et al., 2011). At the end of the experiment, participants were debriefed about the stress induction procedure.

It should be noted that the present stress induction method closely corresponds with the determinants of the human stress response described by Mason (1968), that is, unpredictability, novelty, and uncontrollability.

\section{Stimuli}

Initially, 200 color photographs of complex scenes, depicting distinct meaningful activities, were carefully selected from a commercially available image database. For each picture, we created a unique, one-sentence description of 5-10 words describing the central meaning (gist) of the scene based on the following two criteria: the sentence should be sufficient to identify the photograph and to distinguish it from the other scenes (Adolphs et al., 2001, 2005). Another set of sentences (based on different photographs not used in this study) were created as lures for the memory test. The clarity and distinctness of these short one-sentence written descriptions of pictures was assured in a separate pilot study, in which 10 additional participants judged whether each
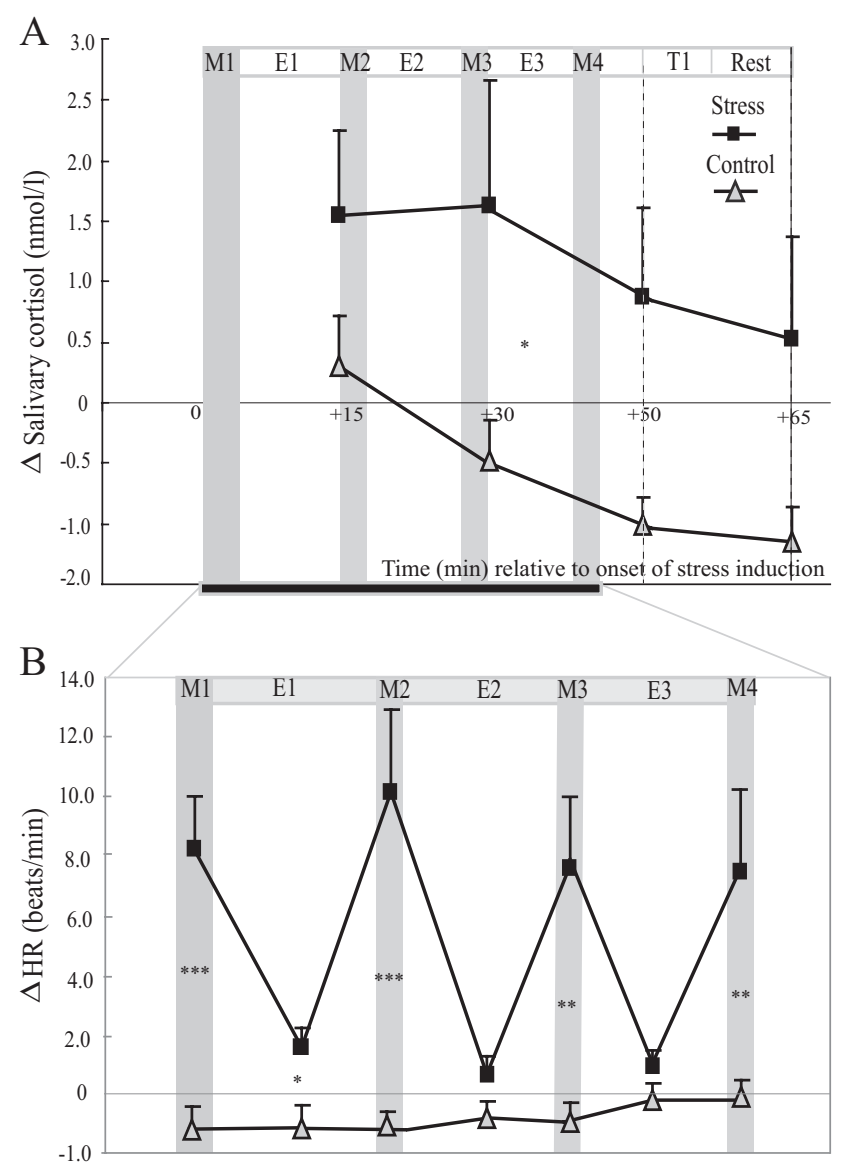

Figure 2. Endocrine and autonomic measures of stress. $A$, Averaged baseline-corrected free salivary cortisol at four time points $(+15,+30,+50$, and +65 min relative to the start of stress induction) for the stress and the control groups. $\boldsymbol{B}$, Averaged baseline-corrected HR during memory encoding sessions (E1-E3) and surrounding movie clips (M1-M4) for the two groups. Error bars represent SEM. Stress, stress group; Control, control group; T1, T1-weighted structural scan; ${ }^{*} p<0.05 ;{ }^{* *} p<0.01 ;{ }^{* * *} p<0.001$.

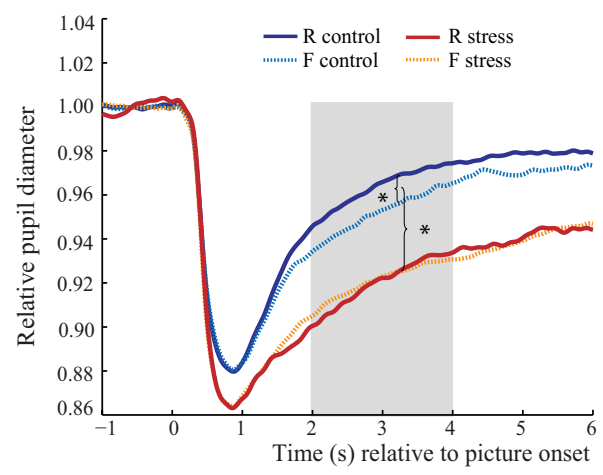

Figure 3. Pupil dilation responses related to memory formation in the stress and the control groups. Pupil dilation responses were normalized with respect to the prestimulus baseline $(-1-0 s)$ and averaged across encoding trials that were later forgotten and those that were later remembered for the two experimental groups. F, Encoding trials later forgotten; $R$, encoding trials later remembered; Stress, stress group; Control, control group; ${ }^{*} p<0.05$.

description sufficiently described the corresponding picture, and unclear descriptions were excluded. The final stimulus set used for the fMRI experiment consisted of 150 scenes (plus 10 extra ones for the training set) with unique, one-sentence descriptions. Luminance of all selected pictures was equalized to ensure reliable pupil dilation measurements. 
Table 2. Overall memory performance in stress induction and control groups

\begin{tabular}{lllll}
\hline & Hit rate & FA rate & $d^{\prime}$ & C-bias \\
\hline Stress $(n=20)$ & $0.74(0.02)$ & $0.30(0.03)$ & $0.89(0.05)$ & $-0.04(0.07)$ \\
Control $(n=20)$ & $0.66(0.02)$ & $0.20(0.03)$ & $0.94(0.05)$ & $0.24(0.07)$ \\
$t(\mathrm{df}=38)$ & $2.97^{* *}$ & $2.30^{*}$ & 0.77 & $-2.76^{* *}$ \\
\hline
\end{tabular}

$\mathrm{FA}$, false alarm; $d^{\prime}$, discrimination index; C-bias, index of response bias; $t$, two-sample $t$ test; df, degree of freedom; ${ }^{* *} p<0.01 ;{ }^{*} p<0.05$.

Memory performance

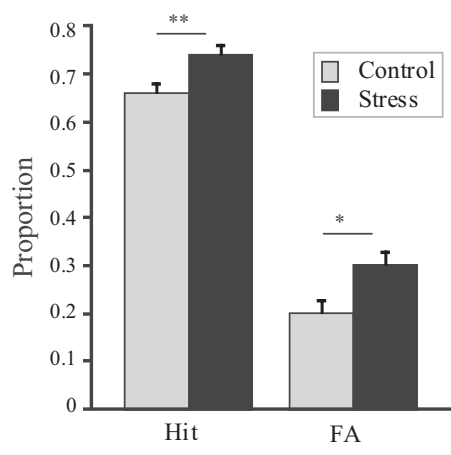

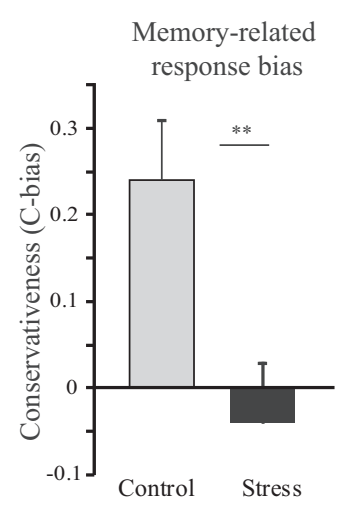

runs of 50 trials each. Within each run, 10 null events of $6 \mathrm{~s}$ duration were intermixed to minimize potential effects of expectation and to optimize contrast-to-noise ratio of event-related fMRI signal. Each run lasted $\sim 12 \mathrm{~min}$. To familiarize participants with the procedure beforehand, they were trained twice using 10 trials that were not used in the actual experiment.

Approximately at the same time on the consecutive day $(\sim 24 \mathrm{~h}$ later, when stress had subsided), participants came back and performed a surprise memory test. Before the start of the memMemory accuracy

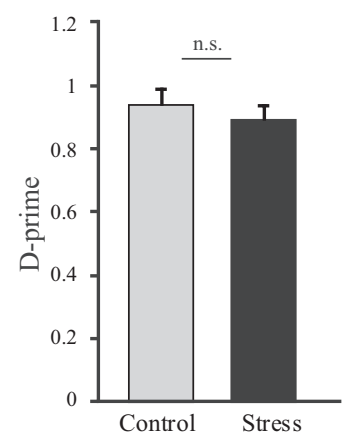
ory test, participants were asked whether they had expected such a test while being scanned the day before. None had expected a memory test. After the test, the experimenter debriefed the participants by explaining the rationale of the surprise memory test. The memory test consisted of 150 written descriptions of studied scenes that were randomly intermixed with 150 descriptions of scenes that were not studied before. Participants were asked to judge whether each description was associated with a scene studied before or not (i.e., "Yes" or "No") and give a confidence rating on a visual analog scale ranging from $0 \%$ to $100 \%$ by moving the cursor via a mouse movement to the appropriate position (Qin et al., 2011). This task was self-paced with a trial duration limited to a range between 2 and $8 \mathrm{~s}$.

It should be noted that we used a memory test for identifying written descriptions of studied scenes rather than a conventional rec-

Figure 4. Memory performance in the stress and control groups. Bar graphs depict hit rate and false alarm rate (left), related response bias as indexed by (-bias or conservativeness (middle), and memory accuracy as indexed by D-prime (right). Hit, hit rate; FA, false alarm rate; ${ }^{* *} p<0.01$.

Table 3. Brain activation associated with the main effect of stress and levels of cortisol

\begin{tabular}{|c|c|c|c|c|}
\hline Brain region & Hemisphere & $\mathrm{BA}$ & $\begin{array}{l}\text { Peak } t \\
\text { value }\end{array}$ & $\begin{array}{l}\text { Coordinates }(x, y, z) \\
\text { (MNI152) }\end{array}$ \\
\hline \multicolumn{5}{|c|}{ Main effect of stress: stress versus control groups } \\
\hline \multirow[t]{2}{*}{ Posterior visual cortex } & $\mathrm{R}$ & 18 & 4.92 & $12,-98,-8$ \\
\hline & $\mathrm{R}$ & 17 & 4.37 & $10,-92,6$ \\
\hline \multirow[t]{2}{*}{$\mathrm{dACC}$ and $\mathrm{mCC}$} & $\mathrm{R}$ & 32 & 4.24 & $6,10,40$ \\
\hline & - & 6 & 4.52 & $0,-10,58$ \\
\hline Striatum & $\mathrm{R}$ & - & 3.94 & $24,4,0$ \\
\hline \multirow[t]{2}{*}{ Insula } & $\mathrm{R}$ & 22 & 3.91 & $54,16,0$ \\
\hline & & & 3.62 & $48,2,2$ \\
\hline \multicolumn{5}{|c|}{ Positive correlation of brain activity with levels of cortisol in the stress group } \\
\hline \multirow[t]{2}{*}{ Thalamus } & $\mathrm{R}$ & - & 7.07 & $22,-18,14$ \\
\hline & $\mathrm{L}$ & & 4.74 & $-16,-12,10$ \\
\hline \multirow[t]{2}{*}{ Insula } & $\mathrm{R}$ & 13 & 6.99 & $34,-18,4$ \\
\hline & L & & 5.48 & $-34,0,12$ \\
\hline \multirow[t]{2}{*}{$\mathrm{mCC}$} & $\mathrm{R}$ & 24 & 6.97 & $6,-10,54$ \\
\hline & $\mathrm{L}$ & & 5.89 & $-14,-12,42$ \\
\hline Striatum & $\mathrm{L}$ & - & 4.90 & $-22,10,10$ \\
\hline \multirow[t]{2}{*}{ Posterior visual cortices } & $\mathrm{R}$ & 18 & 4.89 & $22,-72,-16$ \\
\hline & $\mathrm{L}$ & 19 & 4.53 & $-36,-70,-18$ \\
\hline
\end{tabular}

Only clusters significant at $p<0.05$, corrected at the cluster level, are reported. $\mathrm{dACC}$, Dorsoal anterior cingulate cortex; mCC, middle cingulate cortex; L, left; R, right; BA, Brodmann area; MNI152, Montreal Neurological Institute 152 stereotactic space.

\section{Encoding task and memory test}

The experiment consisted of a study phase on Day 1 (Fig. 1) and a surprise memory test on the consecutive day (Day 2). During the study phase, participants were scanned while performing an incidental encoding task on 150 sequentially and centrally presented photographs (presentation time: $6 \mathrm{~s}$; mean inter trial interval: $6 \mathrm{~s}$, randomly varying from 3 to $9 \mathrm{~s}$ ). Participants were instructed to imagine themselves being in the scene as vividly as possible, and to make a judgment of how much they would like to be present in that scene on a four-point scale (i.e., from "would like it very much" to "would not like it at all"). To ensure that participants did not expect any subsequent memory test, they were told that they were participating in a study on mental imagery with brain imaging and pupillometry. The study phase was divided into three ognition memory test for the following three reasons. First, this paradigm has been shown to assess memory for the gist of an episode (Adolphs et al., 2001, 2005; Qin et al., 2011). Thus, it allowed us to more closely examine the hypothesis of how acute stress leads to a shift of mnemonic processing toward extracting central thematic information, or gist. Second, unlike conventional recognition paradigms, which are dominated by familiarity-based recognition, identification of written descriptions of studied complex scenes without actual perceptual support is most likely more based on recollection. Finally, our paradigm more closely resembles real-life phenomena of eye-witness reports, which typically involve recollection rather than recognition, and therefore optimizes ecological validity.

To accommodate variability of their distribution, confidence ratings of each participant were grouped into four bins with increasing ratings. These four bins corresponded with equal lengths on the visual analog scale in terms of each participant's own distribution of responses. To probe neural correlates truly related to successful memory formation, the fMRI data analysis only focused on trials within the highest of the four confidence level categories compared with trials that were forgotten (i.e., all misses; Table 1). Trials remembered with lower levels of confidence were modeled using a regressor of no interest and were excluded from further analyses. We opted for this method to minimize heterogeneity of memory strength that occurs when trials remembered with different levels of confidence are grouped together. Such an approach has been shown to improve statistical power for identifying neural correlates of successful memory formation (Wagner et al., 1998; Paller and Wagner, 2002; Ranganath et al., 2004; Shrager et al., 2008).

\section{Physiological and psychological measurements of stress}

To monitor the HPA axis response, saliva samples were collected using salivette collection devices (Sarstedt). Salivary sampling consisted of six measurements on Day 1 at $-60,-45,+15,+30,+50$, and +65 min relative to the start of the stress induction procedure. Two saliva samples were collected on Day 2 around memory testing and at time points approximately corresponding with the saliva samples taken at time points +15 and +65 . All samples were stored at $-20^{\circ} \mathrm{C}$ until analysis. Samples were prepared for biochemical analysis by centrifuging at $3000 \mathrm{rpm}$ for 5 min (in the Department of Biopsychology, Trier University Dresden, Germany), which resulted in a clear supernatant of low viscosity. Salivary-free cortisol concentrations were determined using a chemilu- 
minescence assay (CLIA; IBL) with high sensitivity of $0.16 \mathrm{ng} / \mathrm{ml}$. Cortisol data from one participant in the control group were excluded from further analyses because of unexpected residues in the first two samples which precluded reliable cortisol assays.

To increase statistical power for analyses of stress responses, cortisol levels on Day 1 were corrected for baseline differences. We did not use saliva samples taken before stress induction on Day 1 for this baseline correction, because participants were informed in advance to which group they were assigned and thus group differences due to anticipation may have been present in these samples. Instead, after carefully verifying that no remaining group differences were present at this time, we used (averaged) saliva samples from Day 2 for the baseline correction. To incorporate stress-induced changes in cortisol levels measured over time, area under the curve (AUC) with respect to the baseline (obtained from Day 2) was calculated for cortisol levels at all time points after stress induction (i.e., $+15,+30,+50$, and $+65 \mathrm{~min}$ ).

To assess activation of the autonomic nervous system, HR and pupil dilation responses were recorded continuously throughout MRI scanning. HR was recorded by using an MR-compatible BrainAmp MR plus ECG recording system (Brain Products). Eye tracking was performed using an MR-compatible eye-tracking device (MEye Track-LR camera unit; SensoMotoric Instruments) to assess pupil dilation responses. Eyetracking was also used to confirm attentive viewing of movie clips and pictures. Offline analysis for HR included artifact correction and peak detection using Brain Vision Analyzer (Version 1.05) and calculation of HR frequency in Matlab 7.5. Eye pupil data were also analyzed using in-house software implemented in Matlab 7.5, which was based on methods described previously (Siegle et al., 2003; Henckens et al., 2009). Eyeblink artifacts were identified as eye pupil changes occurring too rapidly to represent actual dilation. Blinks were removed from the signal using linear interpolation. The time course of pupil diameter changes (reflecting phasic pupil dilation response) for each trial was normalized to the average $1 \mathrm{~s}$ prestimulus onset baseline. The averaged baseline-corrected pupil diameter within a 2-4 s window during picture presentation was used as response measure. Data from four subjects (two from each group) were excluded from further analyses due to excessive signal artifacts.

In addition, subjective state was assessed on the first day using the positive and negative affect scale (PANAS) questionnaire (Watson et al., 1988) at time points coinciding with collection of saliva samples. Physiological data including HR, and pupil response, as well as psychological data including PANAS and memory performance, were analyzed in SPSS (16.0; SPSS) using repeated-measures ANOVAs and appropriate follow-up $t$ tests. Alpha was set at 0.05 throughout.

fMRI data acquisition. Whole brain $\mathrm{T} 2{ }^{*}$-weighted gradient echo EPI images with BOLD contrast were acquired with a Siemens Tim Trio 3.0T MR scanner using an ascending slice acquisition sequence (37 axial slices; TR, $2.1 \mathrm{~s}$; TE, $25 \mathrm{~ms}$; flip angle, $90^{\circ}$; slice matrix size, $64 \times 64$; slice thickness, $3.0 \mathrm{~mm}$; slice gap, $0.3 \mathrm{~mm}$; FOV, $212 \times 212 \mathrm{~mm}$ ). There were three runs of $12 \mathrm{~min}$ each. High-resolution structural images $(1 \times$ $1 \times 1 \mathrm{~mm})$ were acquired using a T1-weighted three-dimensional magnetization-prepared rapid gradient-echo sequence (TR, $2.3 \mathrm{~s}$; TE, 2.96 $\mathrm{ms}$, flip angle, $8^{\circ}$, FOV , $256 \times 256 \mathrm{~mm}$ ).

$f M R I$ data analysis. Image preprocessing and statistical analysis were performed using SPM5. The first five EPI volumes were discarded to allow for T1 equilibration. Remaining functional images were rigid-body motion corrected, coregistered to the corresponding T1-weighted image, corrected for slice acquisition timing, spatially normalized into a common stereotactic space, resampled into $2 \mathrm{~mm}$ isotropic voxels, and smoothed by convolving with an isotropic 3D Gaussian kernel $(8 \mathrm{~mm})$. The data were statistically analyzed using general linear models and statistical parametric mapping.

To assess transient neural activity related to memory formation, two separate regressors of interest (i.e., encoding trials later remembered with high confidence and those later forgotten; Table 1) were created based on subsequent memory performance, and then convolved with the canonical hemodynamic response function. To avoid potential confounds of group difference in memory-related response bias, the number of trials of each regressor was equalized between the two groups. Remaining trials were modeled as a single regressor of no interest. Additionally, realign-
Table 4. Brain activation associated with successful memory formation across groups (subsequent memory effect: later remembered vs forgotten)

\begin{tabular}{llrll}
\hline Brain region & Hemisphere & BA & $\begin{array}{l}\text { Peakt } \\
\text { value }\end{array}$ & $\begin{array}{l}\text { Coordinates }(x, y, z) \\
\text { (MNI152) }\end{array}$ \\
\hline Inferior frontal gyrus & $\mathrm{L}$ & 45 & 5.43 & $-46,26,6$ \\
MPFC and ACC & & 10 & 4.05 & $-44,44,-2$ \\
& $\mathrm{~L}$ & 8 & 5.64 & $-10,52,40$ \\
Hippocampus & $\mathrm{L}$ & 10 & 4.52 & $-6,54,12$ \\
& $\mathrm{~L}$ & - & $\mathbf{4 . 5 0}$ & $\mathbf{- 3 0 , - 2 6 , - 1 0}$ \\
Angular gyrus and TPJ & $\mathrm{R}$ & - & $\mathbf{4 . 0 2}$ & $\mathbf{2 4 , - 2 4 , - 1 4}$ \\
& $\mathrm{L}$ & 39 & 5.07 & $-48,-60,22$ \\
& & & 3.76 & $-46,-68,40$ \\
Posterior cingulate cortex & $\mathrm{R}$ & 39 & 4.01 & $46,-66,18$ \\
& $\mathrm{~L}$ & 30 & 5.56 & $-6,-52,16$ \\
Cerebellum and brainstem & $\mathrm{L}$ & 31 & 4.26 & $-2,-38,40$ \\
& & - & 4.56 & $-8,-48,-42$ \\
& & & 3.83 & $-4,-34,-40$ \\
\hline
\end{tabular}

Only clusters significant at $p<0.05$, corrected at the cluster level, are reported. Clusters in the medial temporal lob are printed in bold. MPFC, medial prefrontal cortex; ACC, anterior cingulate cortex; TPJ, temporoparietal junction; L, left; R, right; BA, Brodmann area; MNI152, Montreal Neurological Institute 152 stereotactic space.

ment parameters were included to account for movement-related variability. The analyses included high-pass filtering using a cutoff of $1 / 128$ $\mathrm{Hz}$, global intensity normalization, and serial correlations correction using a first-order autoregressive model.

Relevant contrast parameter estimate images were initially generated at the single-subject level, and then submitted to a 2 (group) by 2 (memory) ANOVA for the second-level group analysis treating participants as a random variable. In the whole-brain exploratory analysis, results from the group analysis were initially thresholded at $p<0.001$, uncorrected, and cluster size statistics were used as the test statistic. Unless otherwise specified, only clusters significant at $p<0.05$, corrected (Worsley et al., 1996), are reported. A spherical search region with an $8 \mathrm{~mm}$ radius, centered at the peak voxel of the main effect of memory in the hippocampus, was used for small volume corrections to detect effects of stress on SMEs. To examine regional overlap between the main effect of memory and stress-by-memory interaction, an additional conjunction analysis was performed using the minimum statistic compared with the conjunction-null method in SPM5 (Nichols et al., 2005).

To further characterize patterns of hippocampal and midbrain activity related to memory formation in the two groups, we conducted regions of interest (ROI) analyses on extracted data for these two regions. The ROI in hippocampus was defined by the combination of a hippocampal anatomical mask and an orthogonal contrast (with respect to the interaction effect) associated with the main effect of memory across two groups, while the midbrain mask was defined anatomically. The hippocampal and midbrain masks were defined using the WFU PickAtlas toolbox (Maldjian et al., 2003). Beta values corresponding to conditions of interest were extracted from those ROIs by using MarsBar (Brett et al., 2002) and then subjected to further statistical tests in SPSS.

\section{Results}

\section{Endocrine, autonomic, and psychological measurements of stress}

Baseline-corrected salivary cortisol data are shown in Figure $2 \mathrm{~A}$. A 2-by-4 ANOVA, with group (stress vs control) as betweensubject factor and time (four time points) as within-subject factor revealed main effects of stress induction $\left(F_{(1,37)}=4.72, p<0.05\right.$, with higher cortisol levels in the stress group) and time $\left(F_{(3,50.22)}=\right.$ $4.40, p<0.05)$, but no interaction $(F<1)$. The observed downward trend of cortisol levels over time is most likely due to diurnal rhythm and stress anticipation.

To check whether differences in anticipation of stress induction had led to group differences in cortisol before the start of the experiment, we tested for group differences in the cortisol samples obtained before the start of the experiment on Day 1. Although cortisol levels were numerically higher in the stress group 


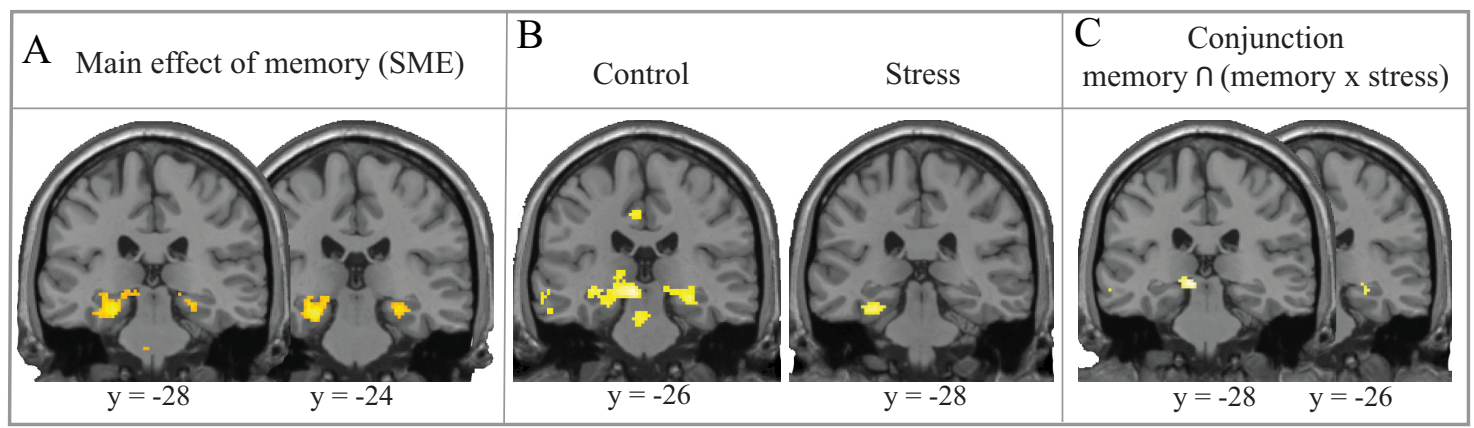

Figure 5. Brain activation related to memory formation and effects of stress induction. Statistical parametric maps are superimposed onto spatially normalized high-resolution single-subject T1-weighted canonical images (thresholded at $p<0.001$, uncorrected, for visualization purposes). $\boldsymbol{A}$, Coronal view of suprathreshold clusters in the medial temporal lobe showing the main effect of memory (SME; remembered vs forgotten). $\boldsymbol{B}$, Coronal view of activation clusters in the medial temporal lobe and the midbrain related to memory formation in the control group (left) and in the parahippocampal cortex in the stress group (right). C, Coronal view of suprathreshold clusters in the conjunction analysis of the main effect of memory and the stress-by-memory interaction. Stress, stress group; Control, control group.

(i.e., average cortisol level and SEM for the two samples before the experiment: $7.92 \pm 0.79$ and $7.32 \pm 1.11$ in the stress group, $6.93 \pm 0.68$ and $5.99 \pm 0.81$ in the control group), this difference did not reach significance $\left(F_{(1,37)}=1.13 p=0.30\right)$. Moreover, no group difference whatsoever was found in cortisol levels on Day 2 (i.e., average cortisol level and SEM for the two baseline samples: $4.63 \pm 0.70$ and $3.17 \pm 0.50$ in the stress group, $4.82 \pm 0.43$ and $3.02 \pm 0.29$ in the control group; difference: $F<1$ ). Thus, the two experimental groups did not differ in cortisol levels at the time of retrieval.

Baseline-corrected HR (Fig. 2B) was averaged separately for three encoding runs and four movie clips. A 2 (group) by 7 (time: four movie clips, three encoding runs) ANOVA was conducted for HR data. A main effect of stress induction was found $\left(F_{(1,38)}=\right.$ 15.97, $p<0.001)$, with increased HR in the stress compared with the control group. The two groups did not differ in $\operatorname{HR}\left(t_{(38)}=\right.$ -1.13 , n.s.) at baseline.

Subjective ratings of negative affect were submitted to a 2 (group) by 4 (time) ANOVA, which revealed a main effect of stress induction $\left(F_{(1,38)}=5.82, p<0.02\right)$, with higher negative affect in the stress induction group.

Altogether, these results confirm that stress induction resulted in activation of the HPA axis and the autonomic nervous system, and led to increased negative affect.

\section{Stress-induced changes in pupil responses related to memory formation}

Averaged pupil dilation responses time-locked to scene picture onsets are shown in Figure 3 as a function of trials later remembered versus forgotten, and separately for the two groups. A 2 (group) by 2 (memory) ANOVA revealed a significant main effect of group $\left(F_{(1,36)}=4.58, p<0.05\right)$ and an interaction effect $\left(F_{(1,36)}=4.23, p<0.05\right)$ within the $2-4$ s time window. The main effect of group indicates that stress induction reduced pupil dilation responses to pictures during encoding in general. Subsequent paired $t$ tests revealed a significant effect of memory in the control $\left(t_{(18)}=2.67, p<0.01\right)$ but not in the stress induction group $\left(t_{(18)}<1\right.$, n.s.), indicating that participants had larger pupil dilation responses to pictures later remembered than to those forgotten in the control condition, but this effect was diminished after stress induction.

\section{Stress-induced changes in memory performance}

Averaged memory performance for the stress and the control groups is listed separately in Table 2 and plotted in Figure 4.
Accuracy in both groups was clearly above chance level $\left(t_{(19)}>\right.$ 25.67, $p<0.001$ ), indicating successful memory formation. Further comparison of the two groups revealed no difference in accuracy $\left(t_{(38)}<1\right.$, n.s. $)$, but did show that there was a more liberal response bias after acute stress $\left(t_{(38)}=2.76, p<0.01\right)$. In other words, acute stress at encoding increased the tendency of participants to endorse items (both studied and unstudied lure items) as having previously been studied.

Moreover, this response bias in the stress group showed a statistical trend for a positive correlation with cortisol elevation $\left(r_{(38)}=0.39, p=0.09\right)$, a positive correlation with averaged $\mathrm{HR}$ increases in response to the stressor $\left(r_{(38)}=0.48, p<0.05\right)$, and a negative correlation with pupil dilation responses to those scenes later remembered $\left(r_{(38)}=-0.67, p<0.01\right)$. This pattern of results indicates that stress-induced activation of the HPA axis, the SNS, and the LC-NE system predicts how liberal the response bias became.

\section{Brain activity showing main effects of stress, memory formation}

First, when comparing neural activity related to the encoding of complex scenes across two groups (main effect of stress), we identified differences of activation in bilateral posterior visual cortices and several other regions, which are thought to comprise a salience processing network (Seeley et al., 2007), in limbic and paralimbic systems including insula, dorsalanterior and middle cingulate cortex, and striatum. Interestingly, we found that cortisol elevation (i.e., AUC) correlated positively with the magnitude of activation in posterior visual cortices, middle cingulate cortex, bilateral thalamus, and insula extending into striatum in the stress group (Table 3 ). In line with the notion of hypervigilant processing under stress, these results indicate that acute stress led to increased activity in a posterior visual network and salience processing network during encoding of scenes. Next, we contrasted encoding trials later remembered with later forgotten (i.e., SME) across the two groups (main effect of memory) and revealed activation of an extended frontal-medial temporal lobe network (Table 4) typically engaged in successful memory formation. This network included clusters in the left inferior and medial PFC and clusters extending from the bilateral hippocampus into parahippocampal regions (Fig. $5 A$ ). 
A

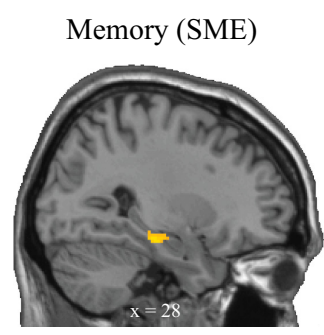

C

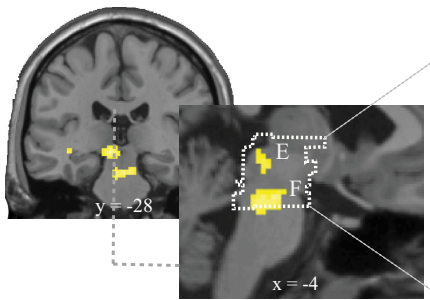

E

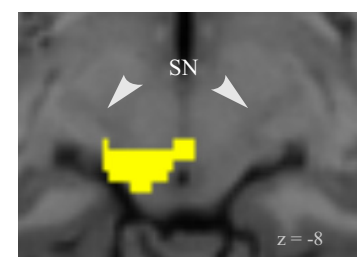

F

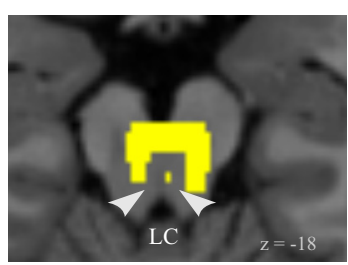

Memory x Stress
B

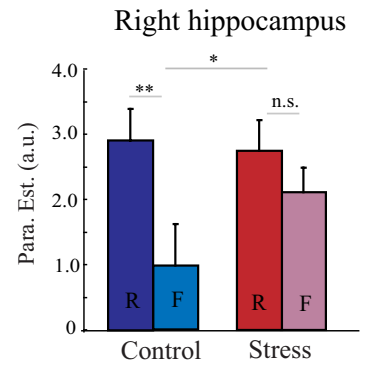

$\mathrm{D}$

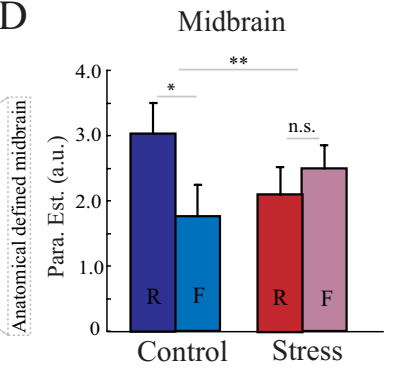

E'

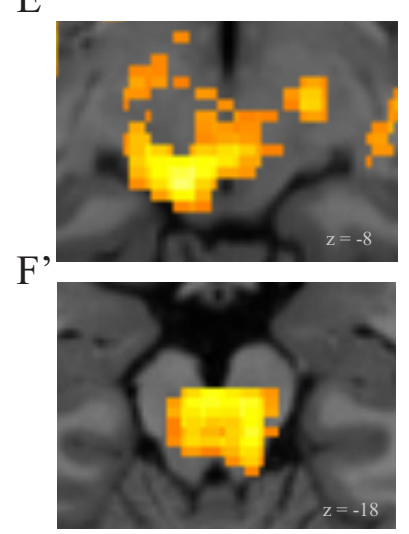

Figure 6. Effects of stress on hippocampal and midbrain activity related to memory formation. Statistical parametric maps are superimposed onto spatially normalized single-subject high-resolution T1-weigted canonical images (thresholded at $p<0.001$, uncorrected, for visualization purposes). $\boldsymbol{A}$, Sagittal view of activation in the right hippocampus associated with the main effect of memory (SME) across the two groups. $\boldsymbol{B}$, Averaged beta values in the right hippocampus representing trials later remembered and forgotten for both groups. C, Coronal (top) and sagittal (bottom) view of suprathreshold clusters in the midbrain for the interaction effect between memory and stress. $\boldsymbol{D}$, Averaged beta values extracted from an anatomically defined midbrain mask representing trials later remembered and forgotten for both groups. $\boldsymbol{E}$, $\boldsymbol{F}$, Transversal view of representative slices for clusters covering the approximate location of midbrain nuclei that are known to be involved in regulation of arousal, homeostatic balance, and vegetative functions, and are also involved in modulation of learning and memory; $E^{\prime}, F^{\prime}$, Same slices as displayed in $E$ and $F$, respectively, but with a less stringent yet acceptable cluster threshold of $p<0.05$, corrected, after thresholding at $p<0.005$, uncorrected, at the voxel level. Localization of these clusters should be interpreted cautiously given limited spatial resolution. Stress, Stress group; Control, control group; $F$, encoding trials later forgotten; $R$, encoding trials later remembered; $\mathrm{SN}$, substantia nigra; $\mathrm{LC}$, locus ceruleus; ${ }^{*} p<0.05 ;{ }^{* *} p<0.01$.

\section{Stress-induced modulations on brain activation related to memory formation}

To examine modulatory effects of stress on neural activity related to memory formation, we conducted a contrast reflecting the interaction between stress and memory by comparing trials later remembered with those later forgotten in the control group versus the stress group. This contrast revealed a large cluster in the midbrain (Fig. 6E,F), likely covering several neuromodulatory nuclei that are known to be involved in regulation of arousal, homeostatic balance, and vegetative functions (Bandler et al.,
Table 5. Suprathreshold clusters associated with the interaction between stress and memory

\begin{tabular}{|c|c|c|c|c|}
\hline Brain region & Hemisphere & $B A$ & $\begin{array}{l}\text { Peak } t \\
\text { value }\end{array}$ & $\begin{array}{l}\text { Coordinates }(x, y, z) \\
\text { (MNI152) }\end{array}$ \\
\hline \multicolumn{5}{|c|}{ Interaction effect: stress (stress vs control) and memory (remembered vs forgotten) } \\
\hline \multirow[t]{3}{*}{ Brainstem/midbrain } & $\mathrm{R}$ & - & 4.03 & $8,-22,-20^{* *}$ \\
\hline & $\mathrm{L}$ & - & 3.98 & $-2,-22,-22^{*}$ \\
\hline & $\mathrm{L}$ & - & 4.01 & $-4,-28,-8^{* *}$ \\
\hline \multirow[t]{2}{*}{ Hippocampus and subiculum } & $\mathbf{L}$ & - & 4.16 & $-8,-28,-6^{*}$ \\
\hline & $\mathbf{R}$ & - & 2.97 & $26,-24,-8^{*}$ \\
\hline Insula & $\mathrm{L}$ & 13 & 4.71 & $-38,-10,-2^{* *}$ \\
\hline \multirow[t]{2}{*}{ Cingulate cortex } & $\mathrm{R}$ & 24 & 4.14 & $2,-16,38^{* *}$ \\
\hline & $\mathrm{L}$ & 31 & 3.99 & $-4,-22,42^{*}$ \\
\hline \multicolumn{5}{|c|}{ Conjunction analysis: stress by memory interaction $\cap$ main effect of memory } \\
\hline \multirow[t]{2}{*}{ Hippocampus and subiculum } & $\mathbf{L}$ & - & 3.30 & $-12,-28,-6^{*}$ \\
\hline & $\mathbf{R}$ & - & 2.81 & $28,-22,-8^{*}$ \\
\hline
\end{tabular}

${ }^{* *} p<0.05$, whole-brain corrected at the cluster level; ${ }^{*} p<0.05$, corrected at the cluster level using a small volume correction. Clusters in the medial temporal lobe are printed in bold. BA, Brodmann area; L, left; R, right; LC, locus coeruleus; SN, substantia nigra; MNI152, Montreal Neu.

2000; Parvizi and Damasio, 2001; Joëls and Baram, 2009), and other clusters in a salience processing network (listed in Table 5). In the medial temporal lobe, we found an interaction in the left hippocampus (likely subiculum) and the right hippocampus. As shown in Figure 5, $A$ and $B$, the hippocampus showed a robust SME in the control group but no reliable effect in the stress group. To ensure actual spatial overlap between this interaction and the main effect of memory, we conducted a conjunction analysis over the two contrasts, which indicated that the right hippocampus and the left subiculum indeed exhibited both the main effect of memory and the interaction effect (Fig. 5C; Table 5).

Moreover, to further characterize the pattern of stressinduced modulations of event-related or phasic hippocampal and midbrain activity, data were extracted and averaged for these two ROIs. Separate 2 (group) by 2 (memory) ANOVAs confirmed the interaction effect in the right hippocampus $\left(F_{(1,38)}=\right.$ $4.26, p<0.05$; Fig. $6 A, B)$ and midbrain $\left(F_{(1,38)}=8.31, p<0.01\right.$; Fig. $6 C, D)$. Separate paired $t$ tests revealed that there were significant SMEs in the right hippocampus $\left(t_{(19)}=3.68, p<0.01\right)$ and midbrain $\left(t_{(19)}=2.57, p<0.02\right)$ in the control group but not in the stress-induction group (both $t_{(19)}<1.80$, n.s.). Together, these results indicate that stress induction reduced SMEs in the hippocampus and midbrain, reflecting less discrimination between items later remembered and forgotten.

\section{Discussion}

The present study investigated how acute psychological stress affects memory formation for neutral information. We observed that acute stress induced a more liberal response bias (i.e., a tendency to endorse items as old) in a memory test involving identification of short written descriptions of scenes encountered $24 \mathrm{~h}$ earlier during stress induction. The strength of this bias correlated with stress-induced changes in cortisol, HR, and pupil dilation. Moreover, acute stress reduced SMEs during memory formation in hippocampus, midbrain, and in pupil dilation. As indicated by stress-induced elevation of cortisol and HR, our stress induction procedure resulted in a shift toward a prolonged state of increased HPA axis and SNS activity. Such states are known to be accompanied by tonic elevation of stress-sensitive catecholamines, regulated by the LC-NE system and midbrain dopaminergic nuclei, and glucocorticoids (de Kloet et al., 2005; Ulrich-Lai and Herman, 2009). We therefore discuss how elevation of these neuromodulators may account for our observed effects of stress on memory formation. 
Acute stress had no effect on overall memory accuracy, but did induce a more liberal response bias. Moreover, individual measures of the amount of stress (i.e., changes in cortisol, HR, and pupil dilation responses) predicted the strength of this bias. These findings are reminiscent of earlier findings of stressinduced elevations of false memory (Payne et al., 2002, 2006, 2007). In these studies, stress induction at the time of encoding led to an increase in the number of false positives for related but novel items during memory testing. Our findings are also consistent with a large amount of literature on the limited reliability of eyewitness reports (Loftus, 1979) and observations of strong subjective remembrance in the absence of increased accuracy for emotional memory (Sharot et al., 2004; Phelps and Sharot, 2008). Notably, effects of stress on retrieval cannot readily explain our findings, because we tested memory after stress had subsided and cortisol levels did not differ anymore. Thus, the more liberal response bias observed after stress likely results from alterations in neurocognitive processing underlying memory formation.

According to influential accounts, emotional arousal or acute stress biases mnemonic operations underlying memory formation toward rapid extraction of central thematic information, or gist (Payne et al., 2002, 2006; Adolphs et al., 2005). Such a shift would have clear survival benefit over encoding details that are not of immediate importance. This alteration can be thought of as increasing the reconstructive nature of such memories: to reduce the amount of detail and focus on a central theme, one would likely need to reconstruct such episodes by reactivating and integrating prior representations of more general categories, i.e., an enhancement of gist-based encoding at the cost of specificity. As a consequence, memory representations encoded under stress may exhibit stronger overlap and thus become more generalized and less discrete, which in turn may explain the observed response bias when probing these memories.

Turning to functional neuroimaging and pupil data, our findings are in line with mechanistic accounts of stress-induced alterations in catecholaminergic neuromodulation of memory-related neural circuits. According to these models, catecholaminergic activity exhibits an inverted U-shaped relationship with efficacy of neurocognitive functioning (Aston-Jones and Cohen, 2005). Intermediate levels of catecholamines are thought to represent an optimal state characterized by selective phasic firing patterns in response to novel and/or salient stimuli. Through such phasic signaling, the hippocampus forms a functional loop with neuromodulatory midbrain nuclei that determines selective processing of novel information and entry into long-term memory (Lisman and Grace, 2005; Harley, 2007). Specifically, novelty signals generated in the hippocampus (Fernández and Tendolkar, 2006) may trigger phasic catecholaminergic activity in neuromodulatory midbrain nuclei, which in turn enhances hippocampal neuroplasticity (Lisman and Grace, 2005; Sara, 2009). Such theoretical models also predict, however, that acute stress would alter the phasic functional coupling of the hippocampus with neuromodulatory nuclei by shifting catecholaminergic systems into a state with reduced phasic, but high tonic background, activity at the right side of the inverted U-shaped curve (Aston-Jones and Cohen, 2005; Arnsten, 2009).

Four findings from the present study lend credence to these notions. First, multiple physiological measures of stress suggest that stress induction lead to tonic elevation of stress-sensitive catecholamines, and the correlation of these stress measures with response bias strength supports an association between activation of stress-sensitive neuromodulatory systems at time of encoding and alterations in memory. Second, the stress-induced enhancement of neural activity during encoding in early visual regions and several regions, which are thought to comprise a salience processing network (Seeley et al., 2007) found in this study and in our previous work (Henckens et al., 2009; Hermans et al., 2011), indicates a state of sensory hypersensitivity under stress. Third, the reduced SMEs in hippocampal, midbrain regions indicate that mnemonic activity in these regions becomes noisier and less selective. Fourth, we found similar reduced effects during memory formation in the event-related pupil dilation response (Fig. 3), a peripheral index of phasic LC-NE system activity (Gilzenrat et al., 2010). Note that the observed reduction of SMEs would also be in line with an alternative interpretation that assumes a drowsy state at the left side of the inverted U-shaped curve with low phasic and low tonic activity (AstonJones and Cohen, 2005). However, our multiple stress response measurements speak strongly against such an interpretation. Therefore, our behavioral, physiological, and neuroimaging findings converge to support the notion that acute stress prompts a hypervigilant state characterized by unselective hypersensitivity to sensory stimuli and noisier signaling during memory formation.

An important question is how such a putative state of hypervigilant processing and noisier signaling can lead to a more positive response bias in the absence of a loss of accuracy. According to recent findings in humans, memory generalization does not rely solely on inferential processes that take place during retrieval, but also relies on integrative and constructive processes already at the time of encoding (Shohamy and Wagner, 2008). Such processes have been associated with functional coupling of the hippocampus and midbrain: phasic dopaminergic firing in response to novel stimuli is thought to trigger reactivations of related or overlapping representations of prior memories and thus result in updating through integration of new information into existing memory (Kumaran and Duzel, 2008; Shohamy and Wagner, 2008; Shohamy et al., 2010). It is therefore possible that the overgeneralization of memory representations that results in a positive response bias is caused by stress-induced tonic activation of this circuit. Yet, memory accuracy for such generalized memory traces could still be preserved under these circumstances because elevated levels of catecholamines and glucocorticoids may generally enhance neural plasticity during encoding (Lisman and Grace, 2005; Harley, 2007) and postencoding consolidation (Joëls et al., 2006; Sara, 2009). Although future research using, for instance, pharmacological manipulations will be necessary to provide more definite answers, our findings suggest that changes in catecholaminergic signaling may play a key role in altering memory formation under stress.

Some caution is warranted when interpreting activations observed in the midbrain. Anatomically, the clusters we found correspond with midbrain sections that are known to contribute to regulating arousal and homeostatic balance in fear and acute stress (Mobbs et al., 2007; Hermans et al., 2011), which parallels our observed vegetative effects such as stress-induced changes in heart rate and pupil responses. These regions are also known as the main sources of various stress-sensitive neuromodulators that are involved in modulating hippocampal functioning underlying learning and memory (Lisman and Grace, 2005; Joëls et al., 2006; Sara, 2009). The involvement of these midbrain nuclei and the hippocampus in memory formation has been reported in many previous neuroimaging studies (Schott et al., 2004, 2006; Wittmann et al., 2005; Sterpenich et al., 2006; Shohamy and Wagner, 2008; Düzel et al., 2009). The observed reduction of SMEs in the midbrain and the hippocampus concur with the proposition that activation of stress-sensitive neuromodulatory systems alter 
the neural processes underlying memory formation. However, given the limitations of conventional fMRI in terms of spatial resolution and the small size of these midbrain nuclei, the precise location of these individual regions is difficult to determine with certainty. Nonetheless, it is conceivable that the observed reduction of SMEs during memory formation results from stressinduced alterations in catecholaminergic signaling, because we found related effects in heart rate and pupil responses. Future research, however, will be necessary to establish this link more directly.

In conclusion, the present study shows that acute stress during encoding results in a more liberal response bias in a memory test for remembrance of neutral information $24 \mathrm{~h}$ later. Moreover, acute stress diminishes SMEs in the hippocampus and midbrain, and in pupil dilation responses (reflecting noradrenergic activity) during memory formation. The reduction of SMEs during encoding and the more liberal response bias at test may be related to a chain of changes in neurocognitive processes underlying memory formation, most likely resulting from stress-induced alterations in catecholaminergic signaling in hippocampus and midbrain circuits. A similar mechanism may account for the low reliability of eyewitness memories for intrinsically neutral information encountered in a stressful context.

\section{References}

Adolphs R, Denburg NL, Tranel D (2001) The amygdala's role in long-term declarative memory for gist and detail. Behav Neurosci 115:983-992.

Adolphs R, Tranel D, Buchanan TW (2005) Amygdala damage impairs emotional memory for gist but not details of complex stimuli. Nat Neurosci 8:512-518.

Arnsten AF (2009) Stress signalling pathways that impair prefrontal cortex structure and function. Nat Rev Neurosci 10:410-422.

Aston-Jones G, Cohen JD (2005) An integrative theory of locus coeruleusnorepinephrine function: adaptive gain and optimal performance. Ann Rev Neurosci 28:403-450.

Bandler R, Keay KA, Floyd N, Price J (2000) Central circuits mediating patterned autonomic activity during active vs. passive emotional coping. Brain Res Bull 53:95-104.

Bradley MM, Miccoli L, Escrig MA, Lang PJ (2008) The pupil as a measure of emotional arousal and autonomic activation. Psychophysiology 45:602-607.

Brett M, Anton JL, Valabregue R, Poline JB (2002) Region of interest analysis using an SPM toolbox. Neuroimage 16:Abstract:497.

Christianson SA (1992) Emotional stress and eyewitness memory: a critical review. Psychol Bull 112:284-309.

de Kloet ER, Joëls M, Holsboer F (2005) Stress and the brain: from adaptation to disease. Nat Rev Neurosci 6:463-475.

Düzel E, Bunzeck N, Guitart-Masip M, Wittmann B, Schott BH, Tobler PN (2009) Functional imaging of the human dopaminergic midbrain. Trends Neurosci 32:321-328.

Fernández G, Tendolkar I (2006) The rhinal cortex: 'gatekeeper' of the declarative memory system. Trends Cogn Sci 10:358-362.

Gilzenrat MS, Nieuwenhuis S, Jepma M, Cohen JD (2010) Pupil diameter tracks changes in control state predicted by the adaptive gain theory of locus coeruleus function. Cogn Affect Behav Neurosci 10:252-269.

Harley CW (2007) Norepinephrine and the dentate gyrus. Prog Brain Res 163:299-318.

Henckens MJ, Hermans EJ, Pu Z, Joëls M, Fernández G (2009) Stressed memories: how acute stress affects memory formation in humans. J Neurosci 29:10111-10119.

Hermans EJ, Putman P, Baas JM, Koppeschaar HP, van Honk J (2006) A single administration of testosterone reduces fear-potentiated startle in humans. Biol Psychiatry 59:872-874.

Hermans EJ, van Marle HJ, Ossewaarde L, Henckens MJ, Qin S, van Kesteren MT, Schoots VC, Cousijn H, Rijpkema M, Oostenveld R, Fernández G (2011) Stress-related noradrenergic activity prompts large-scale neural network reconfiguration. Science 334:1151-1153.

Joëls M, Baram TZ (2009) The neuro-symphony of stress. Nat Rev Neurosci 10:459-466.
Joëls M, Pu Z, Wiegert O, Oitzl MS, Krugers HJ (2006) Learning under stress: how does it work? Trends Cogn Sci 10:152-158.

Kudielka BM, Kirschbaum C (2005) Sex differences in HPA axis responses to stress: a review. Biol Psychol 69:113-132.

Kumaran D, Duzel E (2008) The hippocampus and dopaminergic midbrain: old couple, new insights. Neuron 60:197-200.

Lisman JE, Grace AA (2005) The hippocampal-VTA loop: controlling the entry of information into long-term memory. Neuron 46:703-713.

Loftus EF (1979) Eyewitness testimony. Cambridge, MA: Harvard UP.

Maldjian JA, Laurienti PJ, Kraft RA, Burdette JH (2003) An automated method for neuroanatomic and cytoarchitectonic atlas-based interrogation of fMRI data sets. Neuroimage 19:1233-1239.

Mason JW (1968) A review of psychoendocrine research on the pituitaryadrenal cortical system. Psychosom Med 30 [Suppl]:576-607.

Mobbs D, Petrovic P, Marchant JL, Hassabis D, Weiskopf N, Seymour B, Dolan RJ, Frith CD (2007) When fear is near: threat imminence elicits prefrontal-periaqueductal gray shifts in humans. Science 317:1079-1083.

Nichols T, Brett M, Andersson J, Wager T, Poline JB (2005) Valid conjunction inference with the minimum statistic. Neuroimage 25:653-660.

Ossewaarde L, Hermans EJ, van Wingen GA, Kooijman SC, Johansson IM, Backström T, Fernández G (2010) Neural mechanisms underlying changes in stress-sensitivity across the menstrual cycle. Psychoneuroendocrinology 35:47-55.

Paller KA, Wagner AD (2002) Observing the transformation of experience into memory. Trends Cogn Sci 6:93-102.

Parvizi J, Damasio A (2001) Consciousness and the brainstem. Cognition 79:135-160.

Payne JD, Nadel L, Allen JJ, Thomas KG, Jacobs WJ (2002) The effects of experimentally induced stress on false recognition. Memory 10:1-6.

Payne JD, Jackson ED, Ryan L, Hoscheidt S, Jacobs JW, Nadel L (2006) The impact of stress on neutral and emotional aspects of episodic memory. Memory 14:1-16.

Payne JD, Jackson ED, Hoscheidt S, Ryan L, Jacobs WJ, Nadel L (2007) Stress administered prior to encoding impairs neutral but enhances emotional long-term episodic memories. Learn Mem 14:861-868.

Phelps EA, Sharot T (2008) How (and why) emotion enhances the subjective sense of recollection. Curr Dir Psychol Sci 17:147-152.

Qin S, Hermans EJ, van Marle HJ, Luo J, Fernández G (2009) Acute psychological stress reduces working memory-related activity in the dorsolateral prefrontal cortex. Biol Psychiatry 66:25-32.

Qin S, van Marle HJ, Hermans EJ, Fernández G (2011) Subjective sense of memory strength and the objective amount of information accurately remembered are related to distinct neural correlates at encoding. J Neurosci 31:8920-8927.

Ranganath C, Yonelinas AP, Cohen MX, Dy CJ, Tom SM, D'Esposito M (2004) Dissociable correlates of recollection and familiarity within the medial temporal lobes. Neuropsychologia 42:2-13.

Sara SJ (2009) The locus coeruleus and noradrenergic modulation of cognition. Nat Rev Neurosci 10:211-223.

Schott BH, Sellner DB, Lauer CJ, Habib R, Frey JU, Guderian S, Heinze HJ, Düzel E (2004) Activation of midbrain structures by associative novelty and the formation of explicit memory in humans. Learn Mem 11:383-387.

Schott BH, Seidenbecher CI, Fenker DB, Lauer CJ, Bunzeck N, Bernstein HG, Tischmeyer W, Gundelfinger ED, Heinze HJ, Düzel E (2006) The dopaminergic midbrain participates in human episodic memory formation: evidence from genetic imaging. J Neurosci 26:1407-1417.

Seeley WW, Menon V, Schatzberg AF, Keller J, Glover GH, Kenna H, Reiss AL, Greicius MD (2007) Dissociable intrinsic connectivity networks for salience processing and executive control. J Neurosci 27:2349-2356.

Sharot T, Delgado MR, Phelps EA (2004) How emotion enhances the feeling of remembering. Nat Neurosci 7:1376-1380.

Shohamy D, Wagner AD (2008) Integrating memories in the human brain hippocampal-midbrain encoding of overlapping events. Neuron 60: 378-389.

Shohamy D, Mihalakos P, Chin R, Thomas B, Wagner AD, Tamminga C (2010) Learning and generalization in schizophrenia: effects of disease and antipsychotic drug treatment. Biol Psychiatry 67:926-932.

Shrager Y, Kirwan CB, Squire LR (2008) Activity in both hippocampus and perirhinal cortex predicts the memory strength of subsequently remembered information. Neuron 59:547-553.

Siegle GJ, Steinhauer SR, Stenger VA, Konecky R, Carter CS (2003) Use of 
concurrent pupil dilation assessment to inform interpretation and analysis of fMRI data. Neuroimage 20:114-124.

Sterpenich V, D'Argembeau A, Desseilles M, Balteau E, Albouy G, Vandewalle G, Degueldre C, Luxen A, Collette F, Maquet P (2006) The locus ceruleus is involved in the successful retrieval of emotional memories in humans. J Neurosci 26:7416-7423.

Ulrich-Lai YM, Herman JP (2009) Neural regulation of endocrine and autonomic stress responses. Nat Rev Neurosci 10:397-409.

Valentino RJ, Van Bockstaele E (2008) Convergent regulation of locus coeruleus activity as an adaptive response to stress. Eur J Pharmacol 583:194-203.

van Marle HJ, Hermans EJ, Qin S, Fernández G (2009) From specificity to sensitivity: how acute stress affects amygdala processing of biologically salient stimuli. Biol Psychiatry 66:649-655.
Wagner AD, Schacter DL, Rotte M, Koutstaal W, Maril A, Dale AM, Rosen BR, Buckner RL (1998) Building memories: remembering and forgetting of verbal experiences as predicted by brain activity. Science 281:1188-1191.

Watson D, Clark LA, Tellegen A (1988) Development and validation of brief measures of positive and negative affect: the PANAS scales. J Pers Soc Psychol 54:1063-1070.

Wittmann BC, Schott BH, Guderian S, Frey JU, Heinze HJ, Düzel E (2005) Reward-related FMRI activation of dopaminergic midbrain is associated with enhanced hippocampus-dependent long-term memory formation. Neuron 45:459-467.

Worsley KJ, Marrett S, Neelin P, Vandal AC, Friston KJ, Evans AC (1996) A unified statistical approach for determining significant signals in images of cerebral activation. Hum Brain Mapp 4:58-73. 